\title{
ASSESSMENT OF CEREBRAL OXYGENATION IN ADULT NEUROCRITICAL CARE - WHAT CAN THE NEONATOLOGIST LEARN
}

\author{
E. Keller
}

Neurointensive Care Unit, University Hospital of Zuerich, Zuerich, Switzerland

Early detection and treatment of cerebral ischemia to prevent further neurological damage in stroke patients, is one of the most important issues in Neurocritical Care. Clinical experiences in treatment of patients with severe stroke showed that the available methods to monitor cerebral hemodynamics and oxygenation are insufficient with regard to detection of secondary ischemic events. Our research projects allowed the implementation and exploratory application of advanced near infrared spectroscopy (NIRS) indocyanine green (ICG) dye dilution technology in patients with subarachnoid hemorrhage (SAH). Furthermore, a new probe for combined intracranial pressure (ICP) and CBF monitoring with NIRS and ICG dye dilution was developed. For NIRS a conventional brain tissue probe for ICP monitoring was supplied with two fiber bundles. The new NIRS ICP probe allows combined monitoring of ICP and cerebral hemodynamics in the brain directly, without the influence of extracerebral tissue. Combined monitoring of ICP and NIRS will be of special clinical value in patients with severe hemispheric infarction, SAH and head trauma, already provided with ICP probes and being especially at risk for secondary ischemic brain damage. 